\title{
BASIC PHYSICAL CHEMISTRY FOR THE ATMOSPHERIC SCIENCES
}

\author{
Peter V. Hobbs
}

Peter V. Hobbs est un spécialiste bien connu de la physique de l'atmosphère. Il est l'auteur d'un ouvrage d'introduction à cette discipline publié en 1977 par Academic Press, en collaboration avec John W. Wallace. Lors de la réédition de cet ouvrage en 1990, les auteurs ont inclus un chapitre sur la chimie de l'atmosphère. Comme dans la plupart des livres sur la chimie de l'atmosphère, les notions essentielles de la chimie, et plus précisément ce que l'on appelle la chimie-physique, sont considérées comme connues des lecteurs, ce qui n'est pas le

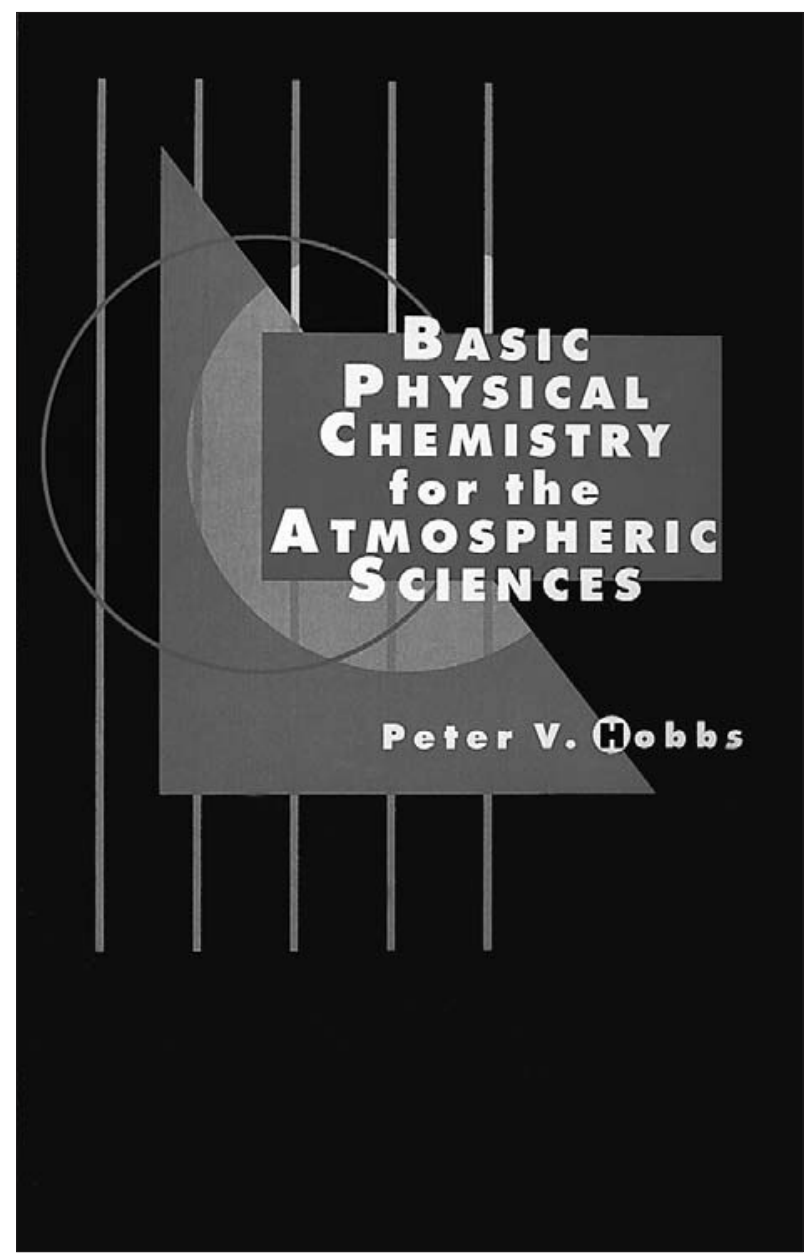
cas pour beaucoup d'entre eux lorsque leur discipline de formation n'est pas la chimie. Les notions de chimie apprises par les physiciens, lors des premières années de l'enseignement supérieur, sont vite oubliées.

La chimie de l'atmosphère est devenue depuis quelques années, avec l'émergence des problèmes de pollution et de modification de la composition et des caractéristiques physico-chimiques de l'air, une composante des sciences de l'atmosphère. L'évolution physico-chimique des constituants gazeux et particulaires doit être prise en compte dans la modélisation de leur transport, dans la prévision d'épisodes de pollution photochimique, etc. Les météorologistes et les physiciens de l'atmosphère doivent remettre à jour leurs connaissances en chimie pour comprendre ce nouvel aspect de leur domaine. Des notions de base en chimie et en chimie de l'atmosphère devraient entrer dans la formation initiale des météorologistes.

Le livre de P. V. Hobbs vient combler une lacune. Il fallait en effet se plonger dans un ouvrage de chimie-physique pour retrouver les notions nécessaires à la compréhension de la chimie de l'atmosphère. Peter V. Hobbs les a rassemblées dans un livre de deux cents pages, dont chaque chapitre peut se lire indépendamment. Le texte est complété par de nombreux exercices, accompagnés de leur solution, et par des exemples pris dans le domaine atmosphérique. Il est accessible à des étudiants de première année de l'enseignement supérieur. Il rendra de grands services à ceux qui sont confrontés aux questions de pollution atmosphérique, ainsi qu'aux mécaniciens des fluides qui ont à modéliser l'évolution des constituants en traces de l'atmosphère.

L'équilibre chimique, la cinétique des réactions chimiques, les lois de la thermodynamique, avec les notions d'enthalpie, d'entropie, d'énergie libre et de constante d'équilibre, sont présentés de façon simple avec le rappel du principe de Le Chatelier sur les systèmes en équilibre, de la relation d'Arrhenius et de l'effet de la température sur les constantes de réaction. Dans l'atmosphère, de nombreuses réactions sont catalytiques. La définition en est rappelée. Les notions de demi-vie, de temps de résidence, de temps de renouvellement d'un constituant en trace, qui sont souvent utilisées par les chimistes de l'atmosphère, sont expliquées.

De nombreuses réactions chimiques se produisent en phase liquide dans les nuages, les précipitations et les brouillards. Là encore, les définitions et notions 
fondamentales qui caractérisent les solutions aqueuses sont présentées de façon synthétique. L'influence du gaz carbonique et des polluants sur le $\mathrm{pH}$ de l'eau en phase liquide dans l'atmosphère est décrite.

Les notions essentielles concernant les réactions d'oxydation et de réduction sont capitales pour comprendre la chimie atmosphérique. Ces réactions se produisent aussi dans les sols, conduisant à des dégagements de constituants en traces dans l'atmosphère. Elles font l'objet d'un chapitre.

Les réactions photochimiques, dont on connaît l'importance dans la stratosphère, mais aussi dans la troposphère, sont présentées avec de nombreux exemples choisis dans les phénomènes atmosphériques.

Le livre de P. V. Hobbs me paraît extrêmement utile. Il concentre dans un volume bien présenté ce que les spécialistes de l'atmosphère doivent maintenant connaître en chimie-physique pour comprendre ce qu'est le milieu atmosphérique, siège de réactions chimiques, physico-chimiques et photochimiques qui sont riches de conséquences sur le bilan radiatif de la planète et sur la biosphère avec l'acidification des précipitations, la formation d'oxydants, etc. Ces connaissances devraient aujourd'hui faire partie de la formation de base aux sciences de l'atmosphère, notamment celle des météorologistes. Je ne peux que recommander l'ouvrage de P. V. Hobbs.

Jacques Fontan

Basic physical chemistry for the atmospheric sciences.

Par P. V. Hobbs.

Cambridge University Press, Cambridge, Royaume-Uni, 1995, 210 p. 